\title{
The Role of Standards for Communicable Disease Prevention and Control in Protecting People's Health and Safety During COVID-19
}

\author{
Lan Feng'; Jingwei Jiang'; Yuan Ma'; Jinxing $\mathrm{Lu}^{1, \ldots}$
}

World Standards Day was established by the International Organization for Standardization (ISO) on October 14 to enhance awareness on the significance of international standardization to respond to the needs of businesses, industries, governments and consumers worldwide. Since its rejoining the ISO in 1978, China held various presentations, symposiums, and commemorative events for World Standards Day in major and medium-sized cities nationwide to publicize the role of standardization in societal development and enhance the awareness of standardization among the people.

The theme for the 51st World Standards Day in this year is "Protecting the planet with standards". Since the coronavirus disease 2019 (COVID-19) pandemic emerged, practitioners in standardization took immediate actions to respond. China's State Standardization Administration, National Health Commission, local governments, and social organizations took emergency actions to formulate and publish a series of relevant national standards, industry standards, local standards, and organization standards for the prevention and control of COVID-19, which played an essential role in regulating and directing efforts in the prevention and control of the outbreak and in protecting the people's health and safety.

\section{REFLECTING ON THE COVID-19 PANDEMIC}

COVID-19 has been the most serious pandemic in the last 100 years and a critical public health event affecting China's development with its fast transmission, wide range of infection, and other significant challenges for prevention and control. The pandemic has highlighted the importance of improving the standards for communicable disease prevention and control and the society's awareness of these measures. President Xi Jinping points out in his recent article, "All-around Improvement of Capability of Prevention, Control, and Governance according to Law to Build a
Sound National Emergency Management System in Public Health" in Qiushi Journal of May 2020, that suggests that the COVID-19 response is "a major test to the state's governance system and capacity." President $\mathrm{Xi}$ also continues to call for practical experience to be summarized and shared to improve the prevention and control standards and the emergency management measures during these critical events. Public health practitioners have since prioritized the in-depth study of how to implement these instructions.

Laws and regulations provide a basis for governance and can regulate the developmental direction of the state and society. Underlying the enforcement of the laws and regulations are a series of more specific and refined standards containing many quantitative requirements, which supplement and extend the legal system. Standardization is the most effective way to realize practical experiences, avoid redundant construction, and enhance efficiency of public services. Building and improving a system of standards is "the last kilometer" in enhancing social governance capacity and accelerating the generation of results for public institutions. Improving communicable disease-related standards can enhance the emergency response capacity in public health to communicable diseases in China.

\section{STANDARDIZING THE RESPONSE TO COMMUNICABLE DISEASES IN CHINA}

Health standards are technical specifications formulated for matters involving human health and healthcare services based on research and practice in order to implement laws, regulations, and policies in healthcare and safeguard human health (1). The National Technical Committee on Health Standards was set up in 1981 to start standardization in healthcare by focusing on preventive medicine. Following this, seven sub-committees were established in the following areas: occupational health, environmental health, food health, diagnosis of 
occupational diseases, radiological protection, diagnosis of diseases from radiation, and school health. With the development of public health and healthcare in China, the Technical Committee on Standards for Communicable Diseases was set up in the 3rd National Technical Committee on Health Standards in 1991, indicating the beginning of standardization in the field of communicable diseases in China. In the 20 years since the establishment of the Technical Committee on Standards for Communicable Diseases, 39 notifiable diseases and emerging and critical communicable diseases have had standards established. The 6th Committee on Health Standards of the Ministry of Health was then set up in March 2008 to reinforce and improve standardization of communicable diseases.

When the 8th Committee on Standards was set up In June 2019, it was renamed the Professional Committee on Communicable Disease Standards of the National Health Standards Committee, and the workplace of the Secretariat changed to China CDC of the National Health Commission (NHC). To date, 52 health standards in communicable diseases have been published, among which 49 are industry standards and 3 are national, and 42 are compulsory and 7 are recommended. There were 45 standards for diagnosis among them, which account for the highest percentage $(91.8 \%)$ and provided a major basis for the prevention, treatment, and monitoring of communicable diseases. The system of standards in communicable diseases has been developed on the basis of years of practical work, international experiences, repeated research, and discussion by the experts. The current framework system of standards for communicable diseases is shown in Figure 1, which provides guidance and specifications for the future formulation of and amendment to standards for communicable diseases.

\section{PROCEDURE OF DEVELOPING HEALTH STANDARDS IN COMMUNICABLE DISEASES IN CHINA}

Health standards in China have been formulated mainly in a government-led manner, which consist mainly of state standards, industry standards, and local standards. The state standards are initiated and published by the State Standardization Administration, the healthcare standards by the National Health Commission, and the local standards by local market regulation administrations. The formulation of a standard system needs to pass a long period of inspection and administrative review and approval. For some standards involving a wide range of technical factors, a long industry chain, and multiple authorities, it could take several years to complete the process from the project proposal to publication.

In recent years, supported by policies encouraging organization standards, significant progress has been made in organizational standards in healthcare, and nearly 40 social organizations represented by Chinese Preventive Medicine Association (CPMA) have carried out development of these standards. Since 2017 when development of organizational standards began, CPMA has reviewed 197 applications for projects of standardization with 126 projects approved. The standards in the field of communicable diseases account for the highest percentage every year.

\section{SHORTCOMINGS IN STANDARDIZATION REVEALED DURING THE COVID-19 PANDEMIC}

Public attention was centralized following the COVID-19 pandemic and related standards came under significant scrutiny: standards for definite diagnosis of the case at the early stages; standards for protective supplies such as masks and suits during the period of rapid spread; concerns about the safe use of imported protection supplies with different standards; safeguard measures and management standards during resumption of work and production as the domestic situation of the outbreak was improved; etc. The State Administration for Market Regulation, NHC, local governments, and social organizations took emergency actions to formulate and publish several series of relevant national standards, healthcare industry standards, local standards, and standards of the organizations, and made widespread efforts to explain the standards to the public. Despite these efforts, considerable shortcomings were revealed in face of the pandemic. Considering the current state of standards for communicable diseases, the following main problems can be identified:

\section{Inadequate Number and Structure of Standards}

While the current industry standards in communicable diseases may cover 39 notifiable diseases, many only consist of a single provision with more than $90 \%$ being standards for diagnosis, which are far from meeting the demands for standards in the prevention and control of communicable diseases. While the Law of the People's Republic of China on 


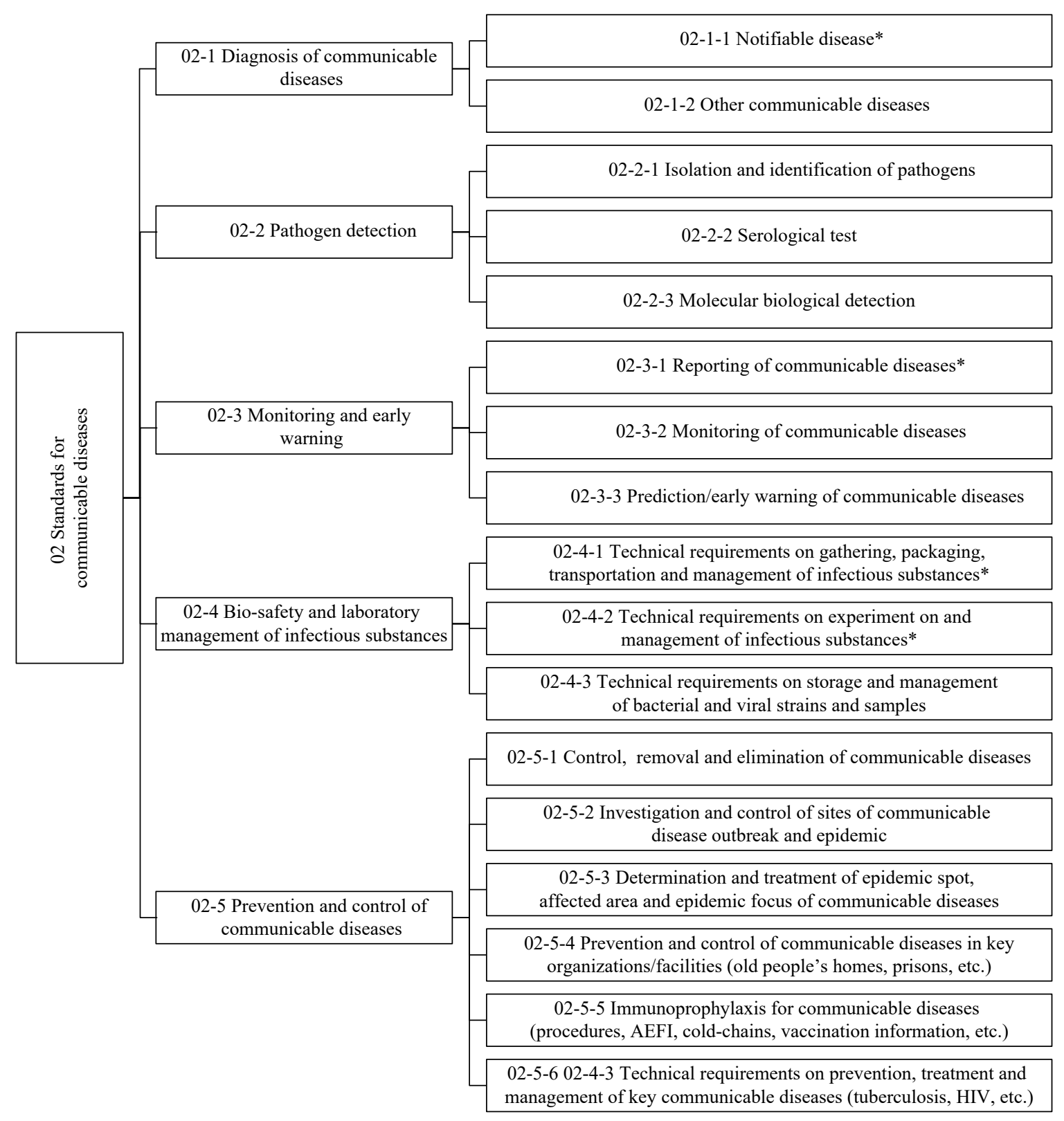

FIGURE 1. Framework system of standards for communicable diseases. Note: those with * are suggested as compulsory standards for the healthcare industry.

Prevention and Control of Infectious Diseases provides clear regulations and requirements on communicable disease monitoring and reporting, there is a lack of detailed, operable monitoring and early warning standards to provide adequate support.

\section{Outdated Standards and Insufficient Publicity and Implementation}

A substantial part of the current standards for communicable diseases are more than 10 years old with several lagging behind the most up-to-date research and technology. Although all the standards over 5 years old were reviewed in 2019, the timely amendment and improvement to these standards is hindered because only a limited number of standards can be amended within a given timeframe. Another issue revealed during pandemic is that many basic-level organizations are not familiar with and/or do not use several published standards, so these standards cannot provide immediate and practical assistance for disease prevention and control at the organizations and neighborhoods mainly due to insufficient publicity and implementation. This situation directly leads to low awareness of the standards and unsatisfactory implementation. 


\section{IMPLICATIONS}

Since the COVID-19 pandemic began, the introduction and guidance of the standardized procedures and related specifications have played an essential role in providing medical supplies, nucleic acid testing, patient treatment and cure, production of masks and protective suits, use of breathing machines and artificial lungs, and the development of vaccines and clinical trials. To achieve the goal of "building a powerful public health system" put forth by President $\mathrm{Xi}$, the shortcomings in the standards for communicable diseases should be addressed so that they can guide, support, and supervise the industry and make a greater contribution to the enhancement of emergency response capacity.

\section{Improving the System by Allowing for Adoption of New Standards and Amendment to existing Standards}

Based on the experiences accumulated from the COVID-19 pandemic, the demands for standards should be further reviewed to optimize the current structure of the standard system for communicable diseases, extend the scope of the standards, and formulate new standards beyond diagnosis to cover communicable disease monitoring, treatment, prevention, result evaluation, and supervision. The strength of the academic societies should be given more authority to regulate and enhance the system of standards that organizations must follow. To practice scientific prevention and control of the outbreak and precise measures, CPMA took urgent measures to collect and approve 20 standards of the organization for prevention and control of the outbreak, which cover rapid detection of the COVID-19 virus, wholegenome sequencing, and other lab detection technologies; sample storage, transportation and other aspects of biosafety; disinfection specifications and environmental sanitation in hospitals, inpatient wards, neighborhoods, schools, mobile hospitals, temporary toilets, and other places during the outbreak; health management during quarantine; etc. Participation of members of the academic committees in standardization and other experts with wide representation should be encouraged during the drafting of the standards, consultation, technical review and other stages, and Internet-based information platforms should be used to promote wide participation, to carry out the principle of "Consultation and Consensus" in standardization.

\section{Reinforcing the Publicity of the Standards}

The impact of the standards relies on their implementation. Publicity, training, and explanation of standardization policies, compilation of standards, and standards for communicable diseases and other related areas should be conducted frequently in multiple forms and dimensions and at multiple levels to enhance standardization awareness, knowledge, and usage.

\section{Promoting the Internationalization of the Standards for Communicable Diseases}

As the COVID-19 pandemic spread worldwide, Chinese experiences in prevention and control were recognized and applied by many counties in international cooperative efforts. Compilation, translation, and publication of standards for communicable diseases in foreign languages should be continued and better funded, and working mechanisms for cooperation between organizations establishing health standards in China, such as the World Health Organization (WHO) and ISO be explored, to promote mutual recognition of the standards for communicable diseases in these international organizations. With standards for communicable diseases as an important starting point for the promotion of construction of the community with a shared future for mankind in terms of healthcare, plans for internationalization of the standards for communicable diseases from China in global affairs and events should be developed to play an active role in formulation of important international standards.

Acknowledgment: The authors would extend their thanks for the guidance from Mr. Lei Suwen, head of Health Standard Division, Chinese Center for Disease Control and Prevention.

doi: $10.46234 / \mathrm{ccdcw} 2020.221$

\# Corresponding author: Jinxing Lu, lujinxing@icdc.cn.

\footnotetext{
National Institute for Communicable Disease Control and Prevention, Chinese Center for Disease Control and Prevention, Beijing, China.
}

Submitted: October 06, 2020; Accepted: October 13, 2020

\section{REFERENCES}

\footnotetext{
1. Huo XJ. Review of development of health standard system in China. China Health Stand Manage 2013;4(1):4-7. http://d.wanfangdata. com.cn/periodical/zgwsbzgl201301002. (In Chinese).
} 